\title{
Serial Ascitic Fluid Analysis - Prognostic Marker for Adverse Outcome in Spontaneous Bacterial Peritonitis in Liver Cirrhosis?
}

\author{
Rahul Pathak, ${ }^{1}$ Annette Von Thun, ${ }^{2}$ Kumar Vikram Singh, ${ }^{3}$ Prem Khadga, ${ }^{4}$ Shashi Sharma ${ }^{5}$
}

${ }^{1}$ Department of Gastroenterology, IOM; ${ }^{2}$ Preventive Medicine Resident, Medical Corps, United States Navy; ${ }^{3} \mathrm{MD}$ resident, Department of Internal Medicine, IOM; ${ }^{4}$ Professor and Head of Department of Gastroenterology, IOM; ${ }^{5}$ Professor and Head of Department of Internal Medicine, IOM, Kathmandu, Nepal

\section{DOI Name}

http://dx.doi.org/10.3126/jaim.v4i1.14174

\section{Keywords}

spontaneous bacterial peritonitis; ascites; prognosis; leukocytes; serial paracentesis

\section{Citation}

Rahul Pathak, Annette Von Thun, Kumar Vikram Singh, Prem Khadga, Shashi Sharma. Serial Ascitic Fluid Analysis-Prognostic Marker for Adverse Outcome in Spontaneous Bacterial Peritonitis in Liver Cirrhosis? Journal of Advances in Internal Medicine 2015;04(01):6-12.

\section{ABSTRACT}

Background and Aims: Cirrhosis is a frequent cause of hospital admissions in Nepal with spontaneous bacterial peritonitis (SBP) being a major determinant of mortality. Limited diagnostic options preclude early risk stratification to improve patient outcomes. We assessed the predictive value of polymorphonuclear leukocyte counts (PMNs) and its association with mortality in hospitalized SBP patients.

Methods: We examined 51 cirrhotic patients diagnosed with SBP in a prospective case-control study admitted to a Nepali tertiary care facility from February 2010 to November 2012. Serial paracentesis and ascitic fluid analysis were performed at admission, 24 , and 48 hours. Receiver operating characteristic (ROC) curves, repeated measures analysis of variance, and logistic regression evaluated ascitic PMNs as predictors of mortality.

Results: Deceased patients demonstrated significantly elevated mean circulating and ascitic PMNs. Ascitic PMNs in the non-survivors were significantly elevated upon admission and at 24 and 48 hours. These differences were significant based on absolute counts, relative differences and trends across time. ROC curves provided diagnostic thresholds $(1600,860$, 500 cells $/ \mathrm{mm}^{3}$ ) distinguishing the two groups upon presentation, at 24 and 48 hours respectively, with high sensitivities (100\%), specificities (82.5-97.5\%), and positive predictive values (52.4-84.6\%). After adjusting for age and case severity, there was a 9.1-fold greater odds of mortality upon admission, for every increase of 500 ascitic PMNs.

Conclusion: In this study of cirrhotic patients with SBP, in-hospital mortality was associated with persistently elevated ascitic PMNs. Ascites PMN thresholds offer a quick, low-tech option to risk stratify patients, prognosticate mortality,

\section{BACKGROUND}

Cirrhosis is a frequent cause of admission to Nepali tertiary healthcare facilities. ${ }^{1}$ One of the major determinants of adverse outcomes in this subset of patients is the presence of spontaneous bacterial peritonitis (SBP) which has been historically associated with high mortality rates. ${ }^{2,3}$ With prompt diagnosis, more aggressive management, and the introduction

\footnotetext{
* Corresponding author

Dr. Rahul Pathak

Department of Gastroenterology, IOM, Kathmandu, Nepal

Email: pathak.drrahul@googlemail.com
} 
of modern broad-spectrum antibiotics, mortality associated with SBP has diminished and is often reported as $20-40 \% .{ }^{4-7}$ Clinical outcomes for some SBP patient populations remain poor despite increases in therapeutic options and availability of more effective antibiotics. ${ }^{4,8,9}$

Unexplainedfever, hypothermia, hypotension, encephalopathy, abdominal pain or unexplained clinical deterioration in cirrhotic patients are considered indications for diagnostic paracentesis. ${ }^{3,8,10}$ Paracentesis has been associated with a short-term survival advantage ${ }^{11}$ and should be performed before administration of antimicrobial therapy to assess for SBP. ${ }^{3,8,10}$ Paracentesis reveals turbid ascitic fluid, an increased leukocyte count, with a predominance of polymorphonuclear leukocyte (PMN) cells. In the majority of cases, cultures of ascites fluid demonstrate a single enteric pathogen. ${ }^{12}$ Although examination of serial ascitic fluid cell counts can demonstrate response to antibiotic therapy, guidelines appear to differ in their recommendations for repeat paracentesis at 48 hours. $3,8,10$ A decrease in the ascites PMN cell count of less than $25 \%$ of the pre-treatment value at 48 hours is generally regarded as an indicator of antibiotic treatment failure. ${ }^{8,10}$ Some authors have challenged this percentage since an increased mortality and SBP relapse has been associated with unresponsiveness to therapy (defined as $>250$ ascitic PMNs $/ \mathrm{mm}^{3}$ at 48 hours) despite significant relative declines in ascitic PMN cells. ${ }^{5,13-16}$

We recently examined the prognostic significance of the Model of End-stage Liver Disease (MELD) scores in a Nepali SBP cohort. ${ }^{17}$ That study demonstrated that deceased patients had a 1.7-fold greater ascites PMN count upon admission than survivors (2301 vs. 1365 cells $/ \mathrm{mm}^{3} ; \mathrm{p}<0.05$ ). The current study assessed cirrhotic patients diagnosed with SBP with serial paracenteses to examine whether differences in serial ascitic fluid was associated with adverse outcomes during hospital admission. The specific objectives were to determine if there were: 1) relevant clinical or laboratory parameters obtained at admission that were associated with increased mortality; 2) significant differences in absolute ascites PMN counts and relative declines in response to therapy between survivor and deceased patients; and 3) if significant PMN thresholds could serve as prognostic indicators to identify and more aggressively manage the high risk cohort.

\section{MATERIALS AND METHODS}

\section{Study design, clinical protocol, and data collection}

The Institute of Medicine is a tertiary care teaching hospital encompassing a broad cross-section of socioeconomic strata of Kathmandu and its surrounding areas. Adult patients (>18 years) consecutively admitted between November 2010 and October 2012 to the gastroenterology department of the Institute of Medicine and diagnosed with cirrhosis and newonset SBP were eligible for inclusion in the study. All patients whose diagnosis of hepatic cirrhosis was confirmed by liver biopsy or ultrasonography were screened for SBP. Study inclusion was independent of cirrhosis etiology. SBP was determined by diagnostic paracentesis upon admission. In accordance with major clinical practice guidelines, patients were considered to have SBP if ascites fluid demonstrated greater than $250 \mathrm{PMNs} / \mathrm{mm}^{3} .3,8,10$ This prospective case-control study represents a secondary analysis of data published previously. ${ }^{17}$

All patients underwent paracentesis initially, within 24 hours, and 48 hours post-admission. Using aseptic technique, approximately 40 milliliters of ascitic fluid was aspirated from each patient and submitted for biochemical and microbiological analysis. Patients diagnosed with SBP upon admission were treated with intravenous cefotaxime. Demographic, clinical and laboratory variables were collected on admission as previously reported. ${ }^{17}$ Mortality was defined as death occurring from any cause during the hospital admission. Exclusion criteria included evidence of secondary peritonitis, or a history of recent antibiotics, gastrointestinal bleed, congestive heart failure or malignancy. ${ }^{17}$ Individuals were also excluded from this analysis if paracenteses were not performed at the ordained time periods per the clinical protocol.

Process review revealed that a large number of the paracenteses performed in the non-survivor group were inconsistent with the timing stipulated by the study protocol. Exclusion of this non-survivor data resulted in a smaller sample size, potentially compromising the power and external validity of the statistical analysis. Some would criticize excluding a large proportion of the data from the deceased group even if there were technical irregularities in the protocol's execution. To address this concern, data were also analyzed using the original cohort of 33 deceased individuals.

\section{Ethical consideration}

The study protocol was approved by the institutional review board committee. All patients gave written informed consent to participate. 


\section{Statistical Analysis}

Patients were classified based upon mortality status during their hospitalization into those that survived and those that died. Categorical variables were analyzed by chi-square tests to assess for differences between survivor and deceased patients. Fisher's Exact test was utilized for those analyses with small samples sizes ( $\leq 5$ individuals). Means, standard deviations (SD), and standard errors (SE) were calculated for continuous variables and were assessed for significance using independent two-tailed Student's t-tests. The non-parametric Mann-Whitney rank sum test was applied to determine significance between the two groups when data for laboratory variables failed to demonstrate normal distribution per Shapiro-Wilk test for normality. Variables found significant by univariate analysis were included in subsequent multivariate analyses. Binary logistic regression was used to assess variables as independent predictors of mortalitywith the final model using variables selected by a backward elimination algorithm.

Receiver operating characteristic (ROC) curves were constructed to determine the diagnostic performance of ascitic leukocytes at the three independent collection times. To assess the serial ascites PMN counts and the interaction of time and mortality, repeated measures analysis of variance (ANOVA) was utilized and Wilks' Lambda post hoc result was reported. Because values for ascitic leukocytes did not meet the ANOVA assumptions of normality and equal variance, data were log transformed and repeated measures ANOVA was performed on transformed data. Data are presented as geometric means with standard error bars calculated on the log scale and transformed to the original units.

Default $p$ values were set a priori at the 0.05 level and 95\% confidence intervals $(\mathrm{Cl})$ were calculated. The Statistical Package for Social Sciences (SPSS, version 15.0, Chicago, II, USA) was used for all statistical analyses.

\section{RESULTS}

A total of 51 patients of the original 73 subjects diagnosed with SBP completed all the necessary laboratory investigations for inclusion in this analysis. The mean $( \pm S D$ ) age of patients in this study was 54.1 ( \pm 10.8 ) years ranging from 28 to 74 years old, with $69 \%$ of patients greater than 50 years of age. The underlying pathology of cirrhosis in our study population was primarily due to alcohol (84\%), with $12 \%$ of cryptogenic etiology and $4 \%$ attributed to hepatitis B. The overall mortality rate in this study was $21.6 \%$.

Demographic data, clinical findings, and laboratory values for the deceased and survivors are presented in Table 1.

Table1: Comparison of demographics, clinical findings, and laboratory values between survivor and deceased patients upon admission.*

\begin{tabular}{|c|c|c|}
\hline & $\begin{array}{l}\text { Survivors } \\
(n=40)\end{array}$ & $\begin{array}{c}\text { Deceased } \\
(n=11)\end{array}$ \\
\hline \multicolumn{3}{|l|}{ Demographics } \\
\hline Age (mean $\pm S D)$ & $54.2( \pm 10.9)$ & $53.5( \pm 11.1)$ \\
\hline Males & $25 \quad(62 \%)$ & $10 \quad(91 \%)$ \\
\hline \multicolumn{3}{|l|}{ Clinical Findings } \\
\hline Abdominal distension & $40(100 \%)$ & 11 (100\%) \\
\hline History of alcohol & $35 \quad(88 \%)$ & $10(91 \%)$ \\
\hline Jaundice & $31(78 \%)$ & $6 \quad(54 \%)$ \\
\hline Icterus & $26 \quad(65 \%)$ & $6 \quad(54 \%)$ \\
\hline Abdominal pain & $23(58 \%)$ & $6 \quad(54 \%)$ \\
\hline Abdominal tenderness & $23(58 \%)$ & $6 \quad(54 \%)$ \\
\hline Fever & $21(52 \%)$ & $7 \quad(64 \%)$ \\
\hline History of GI bleed & $17(42 \%)$ & $4 \quad(36 \%)$ \\
\hline Altered sensorium & $16(40 \%)$ & $5 \quad(46 \%)$ \\
\hline Asterixis & $16(40 \%)$ & $5 \quad(46 \%)$ \\
\hline Oliguria & $13(32 \%)$ & $6 \quad(54 \%)$ \\
\hline Splenomegaly & $10(25 \%)$ & $2(18 \%)$ \\
\hline Hepatomegaly & $2 \quad(5 \%)$ & $0 \quad(0 \%)$ \\
\hline MELD score (mean $\pm S D)$ & $22.9( \pm 5.6)$ & $21.9( \pm 7.4)$ \\
\hline \multicolumn{3}{|l|}{ Laboratory Values } \\
\hline Bilirubin $(\mu \mathrm{mol} / \mathrm{L})$ & $115( \pm 129)$ & $141( \pm 166)$ \\
\hline ALT (U/L) & $83( \pm 57)$ & $89( \pm 56)$ \\
\hline AST (U/L) & $119( \pm 79)$ & $130( \pm 59)$ \\
\hline Albumin (gm/L) & $24.9( \pm 5.2)$ & $25.7( \pm 5.0)$ \\
\hline Urea (mmol/L) & $23.0( \pm 19.7)$ & $18.4( \pm 13.3)$ \\
\hline Creatinine $(\mu \mathrm{mol} / \mathrm{L})$ & $136( \pm 62)$ & $158( \pm 117)$ \\
\hline Prothrombin time (seconds) & $23.1( \pm 5.4)$ & $19.4( \pm 4.0)^{\dagger}$ \\
\hline Hemoglobin (gm/dL) & $9.8( \pm 2.9)$ & $9.5( \pm 2.8)$ \\
\hline Leukocytes (1000 PMNs/mm³) & $7.5( \pm 3.7)$ & $11.4( \pm 4.8)^{\dagger}$ \\
\hline Platelets (1000 cells $/ \mathrm{mm}^{3}$ ) & $95.7( \pm 39.4)$ & $99.6( \pm 28.9)$ \\
\hline
\end{tabular}

* Data presented as counts (\% of cohort) or as mean ( \pm SD).

${ }^{+}$Represents significance of $p<0.05$ as compared to survivor cohort.

Abbreviations: ALT, alanine transaminase; AST, aspartate transaminase; GI, gastrointestinal; MELD, Model of End-stage Liver Disease; PMN, polymorphonuclear leukocytes.

The majority of patients in both groups were found to have abdominal distension, history of alcohol use, jaundice and icterus. There were no significant differences between the two groups for any of the demographic or clinical variables analyzed to include MELD scores which served as a measure 
of liver disease severity. Laboratory values for the survivors and deceased were similar; notable exceptions included significantly elevated mean circulating and ascites PMN counts in the non-survivor group.

Table 2 displays the comparison of ascitic PMN parameters between survivor and deceased patients. Ascitic PMN counts in the deceased cohort were not only elevated upon initial presentation but were also significantly greater at the 24and 48-hour time periods, reflecting 2.4-fold, 2.8-fold, and 3.3-fold greater leukocyte counts, respectively. In addition to the absolute ascitic PMN counts, reductions over time were assessed over the 48-hour period. Survivors demonstrated a greater relative reduction in ascites PMNs from 0 to 24 hours ( $49.5 \%$ vs. $39.4 \%$ ) and from 0 to 48 hours ( $77.8 \%$ vs. $68.0 \%$ ) when expressed as percent of original baseline PMN count ( $p$ $<0.05$ ). Persistent SBP at 48 hours was noted in $100 \%$ of the non-survivors and $71 \%$ in those that survived $(p<0.05)$.

Table 2: Comparison of ascitic PMN parameters between survivor and deceased patients.*

\begin{tabular}{lll}
\hline Ascites Leukocytes & $\begin{array}{c}\text { Survivors } \\
(\mathrm{n}=40)\end{array}$ & $\begin{array}{c}\text { Deceased } \\
(\mathrm{n}=11)\end{array}$ \\
\hline Initial $\left(\mathrm{PMNs} / \mathrm{mm}^{3}\right)$ & $1304( \pm 466)$ & $3139( \pm 1529)^{\dagger}$ \\
At 24 hours $\left(\mathrm{PMNs} / \mathrm{mm}^{3}\right)$ & $681( \pm 381)$ & $1923( \pm 1118)^{\dagger}$ \\
At 48 hours (PMNs/mm $)$ & $238( \pm 122)$ & $932( \pm 281)^{\dagger}$ \\
Average 24-hour decline $(\%)$ & $49.5( \pm 15.4)$ & $39.4( \pm 12.9)^{\dagger}$ \\
Average 48-hour decline $(\%)$ & $77.8( \pm 7.9)$ & $68.0( \pm 7.3)^{\dagger}$ \\
Persistent SBP at 48 hours ${ }^{\ddagger}$ & $27(71 \%)$ & $11(100 \%)^{\dagger}$ \\
\hline
\end{tabular}

* Data presented as mean ( $\pm S D$ ) unless otherwise specified.

${ }^{+}$Represents significance of $p<0.05$ as compared to survivor cohort.

${ }^{\ddagger}$ As defined by $>250 \mathrm{PMNs} / \mathrm{mm}^{3}$; data presented as counts $(\%$ of cohort).

Abbreviations: PMN, polymorphonuclear leukocytes; SBP, spontaneous bacterial peritonitis.

Using ANOVA with repeated measures, effects between the two groups and across time periods were significant $(p<$ 0.001 ) before and after logarithmic transformation of the serial PMN counts. Additionally, progressive trends between the two groups were found to differ significantly (see Figure $1)$, reflecting dissimilarities in their response over time ( $p=$ 0.006). Thus, although the non-survivors displayed greater initial ascitic PMN counts with greater absolute decreases in ascites leukocytes, there was a diminished relative decline over the 48-hour period as compared to the survivors.

ROC curves were constructed at each of the three independent collection timesto determine whether ascitic PMN counts could serve as potential indicators of mortality. The area under the curves for the three time points varied from 0.95 to 0.99 , indicating that all three analyses demonstrated robustness in their predictive value $(p<0.0001)$.

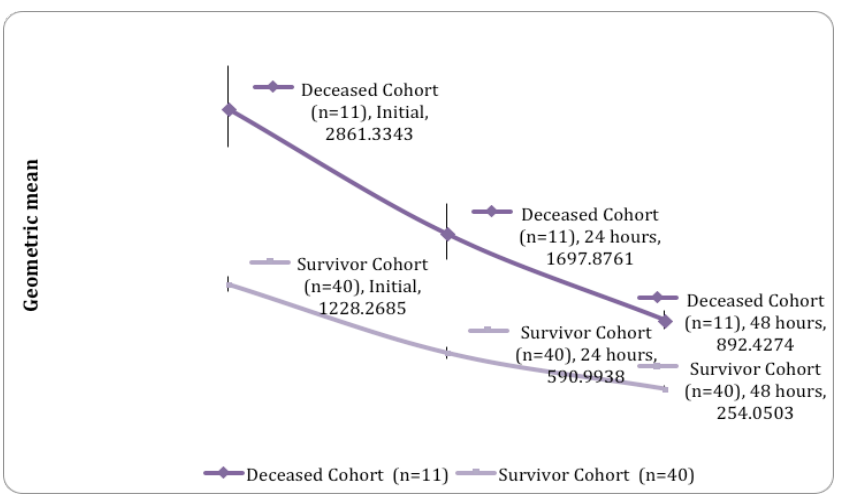

Figure 1: Ascites polymorphonuclear leukocytes counts in deceased and survivor patients upon initial presentation, at 24 hours, and 48 hours after hospital admission.*

* Ascites polymorphonuclear leukocytes counts expressed as geometric means ( $\pm S E$ ). Change of ascitic polymorphonuclear leukocytes over time differed significantly between deceased and survivor cohorts ( $p=0.006$, cohort by time interaction), within individual cohorts $(p<0.001)$, and at each time point $(p<0.001)$.

Ascitic PMN thresholds were established to maximize sensitivity in predicting mortality and are presented in Table 3. Ascites PMN counts $>1600$ cells $/ \mathrm{mm}^{3}$ upon initial presentation, $>860$ cells $/ \mathrm{mm}^{3}$ at 24 hours, and $>500$ cells $/ \mathrm{mm}^{3}$ at 48 hours had a $100 \%$ sensitivity as predictors of mortality with specificities ranging from $82.5 \%$ to $97.5 \%$. Positive predictive values at these three time points varied from $52.4 \%$ at 0 hours to $84.6 \%$ at 48 hours. Dotplots displaying the serial ascites leukocyte counts for the two groups with the prognostic thresholds for each time period are displayed in Figure 2.

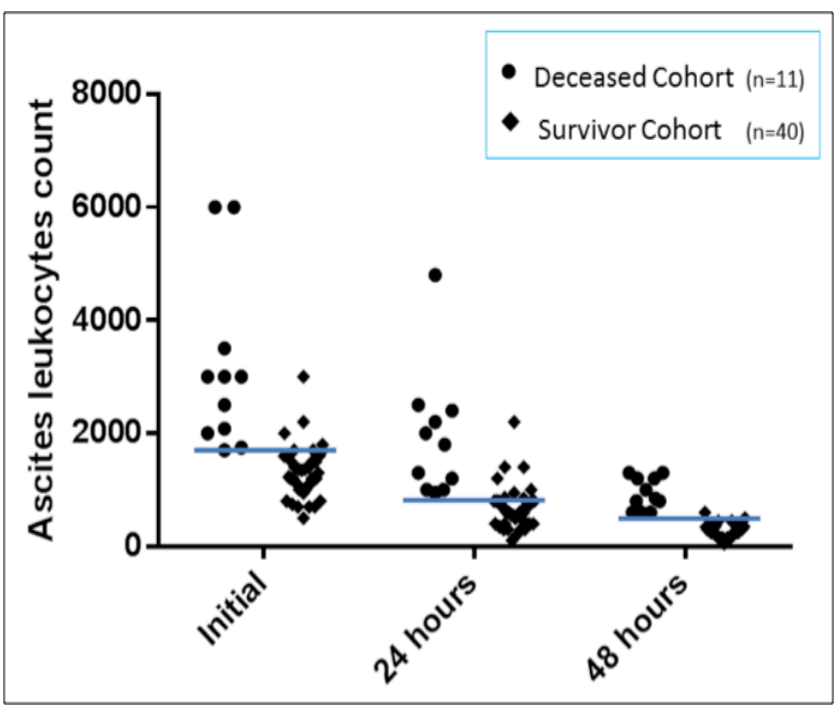

Figure 2: Ascites polymorphonuclear leukocyte counts and prognostic thresholds* in deceased and survivor patients upon initial presentation, at 24 hours, and 48 hours after 
hospital admission.

* Prognostic thresholds, indicated by horizontal bars, were assessed by independent ROC curves constructed at each time point and were determined to be $1600 \mathrm{PMNs} / \mathrm{mm}^{3}$ at initial presentation, $860 \mathrm{PMNs} / \mathrm{mm}^{3}$ at 24 hours, and $500 \mathrm{PMNs} / \mathrm{mm}^{3}$ at 48 hours.

Abbreviations: PMN, polymorphonuclear leukocytes.

Ascites PMNs counts at 0 and 24 hours were found to be independent determinants of in-hospital mortality. An increase of 500 PMNs in the ascites fluid was associated with 5 -fold greater odds of mortality at admission and at 24 hours (Table 3). Despite being the best predictor of mortality, the odds ratio at 48 hours could not be estimated because of the lack of overlap between survivors and non-survivors.

Table 3: Performance characteristics of ascitic polymorphonuclear leukocyte thresholds performed upon initial presentation, at 24 hours, and 48 hours after hospital admission.

\begin{tabular}{|c|c|c|c|c|c|}
\hline $\begin{array}{l}\text { Time } \\
\text { Point }\end{array}$ & $\begin{array}{c}\text { Threshold } \\
\text { (PMNs/ } \\
\mathrm{mm}^{3} \text { ) }\end{array}$ & Sensitivity & Specificity & $\begin{array}{c}\text { Positive } \\
\text { Predictive } \\
\text { Value }\end{array}$ & $\begin{array}{c}\text { Odds Ratio* } \\
\text { per } \\
500 \text { PMNs }\end{array}$ \\
\hline Initial & $>1600$ & $\begin{array}{c}100 \% \\
(71.5-100.0)\end{array}$ & $\begin{array}{c}82.5 \% \\
(67.2-92.7)\end{array}$ & $\begin{array}{c}52.4 \% \\
(32.4-71.7)\end{array}$ & $\begin{array}{c}5.02 \\
(1.85-13.65)\end{array}$ \\
\hline $\begin{array}{l}24 \\
\text { hours }\end{array}$ & $>860$ & $\begin{array}{c}100 \% \\
(71.5-100.0)\end{array}$ & $\begin{array}{c}85.0 \% \\
(70.2-94.3)\end{array}$ & $\begin{array}{c}61.1 \% \\
(38.6-79.7)\end{array}$ & $\begin{array}{c}5.13 \\
(1.91-13.81)\end{array}$ \\
\hline $\begin{array}{l}48 \\
\text { hours }\end{array}$ & $>500$ & $\begin{array}{c}100 \% \\
(71.5-100.0)\end{array}$ & $\begin{array}{c}97.5 \% \\
(86.8-99.9)\end{array}$ & $\begin{array}{c}84.6 \% \\
(57.8-95.7)\end{array}$ & $N / A^{+}$ \\
\hline
\end{tabular}

* Odds ratio not adjusted for other factors. $p<0.002$

${ }^{+}$Although the best predictor of mortality, the odds ratio at 48 hours could not be estimated because of the lackof overlap between survivors and non-survivors.

Abbreviations: PMN, polymorphonuclear leukocytes.

Using backwards elimination by the likelihood ratio test, ascitic PMN count upon admission was determined to be the variable most strongly associated with mortality in the regression model. The odds ratio for this initial paracentesis was 9.07 per every 500 ascitic PMNs (95\% Cl: 1.83, 44.92; $\mathrm{p}=0.007$ ) after adjusting for age and MELD score. CirculatingPMNs were also considered significant in univariate and multivariate analyses, but could not be used in conjunction with ascitic PMN variables in the regression model. After adjustment for age and MELD score, there was a 4.3-fold greater odds of death for every 5000 increase in circulating PMNs (95\% Cl: 1.42, 12.97; $\mathrm{p}=0.01)$.

To address the concern of the study's technical irregularities, the analysis was repeated using the original cohort of 33 deceased individuals (data not shown). There were minimal differences in clinical findings and laboratory values between the survivors and deceased. When the entire population was included in the ascites leukocyte analysis, similar means, declining trends, and prognostic thresholds (1725, 905, 550 vice $1600,860,500$ cells $/ \mathrm{mm}^{3}$ ) were observed. Despite the larger sample size, there were greater variances and less reliability per area under the ROC curves (0.66-0.70).

\section{DISCUSSION}

This study examined the role of serial ascitic PMN counts as a potential prognostic indicator for survivorship in patients with SBP at a Nepali tertiary healthcare facility. Despite comparable demographic, clinical and laboratory variables, the nonsurvivors in our study presented with greater circulating and ascites PMN cells, demonstrated persistent SBP infection, and sustained elevated ascitic PMN counts as compared to their survivor counterparts. These differences were significant based on absolute ascites PMN counts, relative differences and trends across time.

Numerous studies have examined clinical predictors of adverse outcome for cirrhotic patients who develop SBP. High MELD and MELD-derived scores, ${ }^{17-23}$ a history of gastrointestinal bleeding, , 24,25 evidence of impaired renal function, 4,7,23,26,27 and alterations in albumin levels ${ }^{5,24}$ have been among the clinically significant risk indicators. Studies have demonstrated that persistent SBP and a lack of microbiological response is an independent risk factor for SBP-related mortality. ${ }^{9,13,16,18,26}$ Healthcare-acquired infections, $\beta$-lactamase resistance or inadequate antibiotic coverage have also been associated with greater mortality in SBP patients. ${ }^{4,18,26,28-32}$

Prior recommendations have advocated a $25 \%$ decline in ascites PMN counts to reflect a favorable response to therapy. ${ }^{8,10}$ In the current study, average 48-hour declines in ascites PMNs were $68 \%$ in the deceased group, reinforcing the findings that relative declines may be less important than absolute counts in determining outcomes., ${ }^{5,15}$ Other studies have also documented an increased mortality in their nonsurvivor subpopulations, reporting 66-67\% decreases in ascites PMN counts at 48 hours as compared to $92-93 \%$ ascitic PMN declines in their survivor cohorts. ${ }^{14,15}$ Thus, a percent decrease does not appear to be a suitable indicator of mortality. An ascitic PMN count $<250$ cells $/ \mathrm{mm}^{3}$ has been endorsed as a favorable response to therapy at 48 hours and as an endpoint for antimicrobial therapy. Our results suggest that failure to attain an ascitic PMN count $<500$ cells $/ \mathrm{mm}^{3}$ at 48 hours 
carries a greater risk of death, with a positive predictive value of $84.6 \%$.

Perhaps the most important contribution of this study is the identification of discrete diagnostic thresholds that could be utilized to risk stratify patients upon initial presentation and within the first few days of admission. Specifically, our study found that patients $\mathrm{w} />1600 \mathrm{PMNs} / \mathrm{mm}^{3}$ at admission, $>860$ $\mathrm{PMNs} / \mathrm{mm}^{3}$ at 24 hours, and $>500 \mathrm{PMNs} / \mathrm{mm}^{3}$ at 48 hours were more likely to suffer a poor prognosis. Other studies have noted elevated circulating, $6,7,22,30$ or ascites 7,9,15,16,28,33${ }^{35} \mathrm{PMN}$ counts as a risk factor for mortality. Although a small study, Lipka et al. (1998) noted increasing mortality associated with increasing ascitic PMN strata $(<500,500-1000$, and $>1000$ PMNs $/ \mathrm{mm}^{3}$ ), but these authors did not develop ascitic PMN thresholds to predict mortality per se.

We believe our study to be the first to propose specific ascites PMN prognostic thresholds at 0,24 and 48 hours after admission to risk stratify patients and guide patient care. The ROC curves demonstrated reliability and robustness in their predictive value with sensitivities of $100 \%$ and specificities from $82.5 \%$ to $97.5 \%$. These ascites leukocyte thresholds can be utilized to prognosticate mortality and guide patient care. Patients identified to be at high risk upon admission (e.g., ascitic PMN count $>1600$ cells $/ \mathrm{mm}^{3}$ ) could be triaged to receive more aggressive management and may be more appropriate candidates for the judicious administration of adjuvant albumin in our limited resource setting. ${ }^{36-38}$ Subsequent paracenteses could be performed at 48 hours to determine if patients are responding adequately to antimicrobial therapy. If failing to respond, patients' antibiotic regimens can be altered to provide broader coverage or target antibioticresistant organisms. This is particularly important given concerns about increasing prevalence of antibiotic resistance, healthcare-acquired infections and a greater predominance of gram positive organisms that may not be covered by first-line or empiric antibiotics. . $^{9,28,31,35,39-42}$

Development of a quick and easy prognostic tool would provide a significant advantage in preemptively risk stratifying patients upon admission, rather than waiting for culture results, clinical deterioration or antibiotic failure. Unlike some more recent biochemical markers that are being explored as prognostic indicators (e.g., C-reactive protein, interleukin-6, interleukin-22, tumor necrosis factor- $\alpha),{ }^{40,43-45}$ assessing ascites PMN counts is a low-tech and relatively inexpensive diagnostic test that has particular appeal in this low-resource setting. In combination with the MELD score, the ascites PMN count and response could potentially serve as a valuable prognostic tool, 4,22,46 although there may be some settings in which the rise in ascites PMNs may be attenuated. ${ }^{28,32,35,46}$ Recently, Ariza et al. (2013) reported that ascitic PMN counts may be influenced by nosocomial acquisition, the causative microorganisms, and the severity of the underlying liver pathology of the cirrhotic patients-which may complicate the establishment of prognostic thresholds.

Besides its small size, the current study has several potential limitations. Self-prescribing is a common practice in Nepal, so the over-the-counter use of antibiotic therapy, immunosuppressive medications, or proton-pump inhibitors may not have been adequately addressed in our study.Survival was confined to observations made while patients were admitted to the hospital and was not assessed longitudinally for outcomes. Unintentional confounding may have potentially been introduced by the observational design of our study. Additionally, no microorganism data was collected. The relative prevalence of gram positive or cephalosporin-resistant organisms is unknown in our SBP population, and our findings may potentially represent the unique microbiological ecology of our hospital and patient population.

Although larger prospective clinical investigations are needed to validate ascites PMN thresholds, managing SBP by risk stratifying patients upon admission and performing a repeat paracentesis at 48 hours for those at high risk has many advantages particularly in low-resource settings. Cultures frequently take more than 48 hours to become positive. Ascites fluid cultures are often negative or not obtained, thus an early prognostication tool is imperative. More discerning use of repeated paracenteses would reduce unnecessary procedures, costs, nosocomial infections and iatrogenesis. High risk patients can be easily identified initially and more aggressively managed. Based on patients' response to therapy, additional cultures can be judiciously ordered, antibiotic coverage selectively expanded, and patient care appropriately escalated. In addition to larger studies to ascertain PMN diagnostic thresholds, further investigations could explore the etiology of persistent SBP and characterize the pathogenic microorganisms involved in our patient population.

\section{CONCLUSIONS}

This study demonstrated that in-hospital mortality was associated with persistently elevated circulating and ascitic PMN counts in patients with cirrhosis and SBP. Ascitic PMNs 
in the non-survivors were not only elevated upon initial presentation but were also significantly elevated at 24 and 48 hours indicating a poor response to antimicrobial therapy. The differences found between the two subpopulations were significant based on absolute leukocyte counts, relative differences and trends across time. Diagnostic thresholds were defined and determined to be robust and reliable in

\section{REFERENCE}

1. Edelman DA, Sugawa C. Lower gastrointestinal bleeding: a review. Surg Endosc 2007;21:514-20.

2. Barnert J, Messmann H. Management of lower gastrointestinal tract bleeding. Best Pract Res Clin Gastroenterol 2008;22: 295-312.

3. Hreinsson JP, Gumundsson S, Kalaitzakis E, et al. Lower gastrointestinal bleeding: incidence, etiology, and outcomes in a population-based setting. Eur $J$ Gastroenterol Hepatol 2013;25:37-43.

4. Strate LL. Lower GI bleeding: epidemiology and diagnosis. Gastroenterol Clin North Am 2005;34:643-64.

5. Lanas A, Garcia-Rodriguez LA, Polo-Tomas M, et al. Time trends and impact of upper and lower gastrointestinal bleeding and perforation in clinical practice. Am J Gastroenterol 2009; 104:1633-41.

6. Zuckerman GR, Prakash C. Acute lower intestinal bleeding. Part II: etiology, therapy, and outcomes. Gastrointest Endosc 1999; 49:228.

7. Korkis $\mathrm{AM}, \mathrm{McDougall} \mathrm{CJ}$. Rectal bleeding in patients less than 50 years of age. Dig Dis Sci 1995; 40:1520.

8. Zuccaro G Jr. Management of the adult patient with acute lower gastrointestinal bleeding. American College of Gastroenterology. PracticeParameters Committee. Am J Gastroenterol 1998;93:1202-8.

9. Eisen $\mathrm{GM}$, Dominitz $\mathrm{JA}$, Faigel $\mathrm{DO}$, et al. Anannotated algorithmic approach to acute lower gastrointestinalbleeding. Gastrointest Endosc 2001;53:859-63.

10. Jensen DM, Machicado GA. Colonoscopy for diagnosis and treatment of severe lower gastrointestinal bleeding. Routine outcomes and cost analysis. Gastrointest Endosc Clin N Am 1997;7:477-98.

11. Kovacs TO, Jensen DM. Recent advances in the endoscopic diagnosis and therapy of upper gastrointestinal, small intestinal, and colonicbleeding. Med Clin North Am 2002;86:1319-56.

12. Bai $Y$, Peng J, Gao J, et al. Epidemiology of lower distinguishing those with a survival advantage upon admission, at 24 and 48 hours. Ascites PMN thresholds may be utilized to risk stratify patients and guide patient care, and is particularly suited for low-resourced settings. Additional prospective studies with larger sample sizes are needed to validate our findings of using ascites leukocytes as prognostic indicators and in ascertaining appropriate diagnostic thresholds.

gastrointestinal bleeding in China: Single-center series and systematic analysis of Chinese literature with 53,951 patients. J Gastroenterol Hepatol 2011;26:678-82.

13. Anand AC, Patnaik PK, Bhalla VP, et al. Massive lower intestinal bleeding--a decade of experience. Trop Gastroenterol 2001; 22:131-4.

14. Barnert J, Messmann H. Diagnosis and management of lower gastrointestinal bleeding. Nat Rev Gastroenterol Hepatol 2009;6:637-46.

15. Fernández Alonso C, García Lamberechts EJ, Fuentes Ferrer M, et al. Management of lower gastro-intestinal bleeding in the emergency department short-stay unit. Emergencias 2010;22:269-74.

16. Tan BK, Tsang CB, Nyam DC, et al. Management of acute bleeding per rectum. Asian J Surg 2004;27:32-8.

17 .Shennak MM, Tarawneh MM. Pattern of colonic disease in lower gastrointestinal bleeding in Jordanian patients: a prospective colonoscopic study. Dis Colon Rectum 1997;40:208-14.

18. Strate LL, Syngal S. Timing of colonoscopy: impact on length of hospital stay in patients with acute lower intestinal bleeding. Am J Gastroenterol 2003;98:317-22.

19. Schmulewitz N, Fisher DA, Rockey DC. Early colonoscopy for acute lower GI bleeding predicts shorter hospital stay: a retrospective study of experience in a single centre. Gastrointest Endosc 2003;58:841-6.

20. U.S. Preventive Services Task Force. Screening for colorectal cancer: U.S. Preventive Services Task Force Recommendation Statement. Ann Intern Med 2008;149:627-37.

21. Rex DK, Johnson DA, Anderson JC, et al. American College of Gastroenterology guidelines for colorectal cancer screening 2009. Am J Gastroenterol. 2009;104:739-50.

22. Levin B, Lieberman DA, McFarland B, et al. Screening and surveillance for the early detection of colorectal cancer and adenomatous polyps, 2008: a joint guideline from the American Cancer Society, the US Multi-Society Task Force on Colorectal Cancer, and the American College of Radiology. CA Cancer J Clin. 2008;58:130-60. 